\title{
苹果树修剪仿真的框架设计 修剪反应图及其评价的自动生成
}

\author{
俞元中 吴贞利 董 明 孟令杰
}

（安省计萛中心，合肥）

关词苹果树、修剪、仿真(模拟)

在各类果树中苹果树的修技术难度大，相应的理论研究和技术措施常居领先地位. 本 文把单株金冠苹果树作为研究对象,将其分解为骨架和枝组, 以结果枝组的修韵反应和评价为 例,论述对其进行仿真的框架设计思想与软件研制情况.

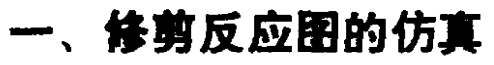

修留技术难以掌握,其重要原因在于修势后的反应无法当场看到. 把次年、多年后的情况 想象出来，需要有相当的理论基础和丰富的实践经验. 利用计算机仿真技术将韵后的枝芽生 成图显示出来,就能为修剪理论研究、修望技术的掌握与改革提供一种先进、有效的手段.

我们通过编制有关程序, 设计了一把“的刀”, 只要在键盘上做些简单操作, 就能使它全屏

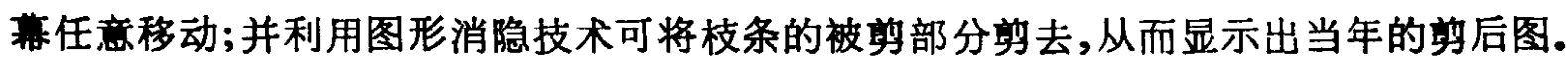

尊口位置 $R J K_{i 0}$ 可由计算机自动识别(图 1).

$R J K_{i 0}=\left\|P_{1} P\right\| /\left\|P_{1} P_{2}\right\|$ 并由此比值可确定是哪种类型的尊法.

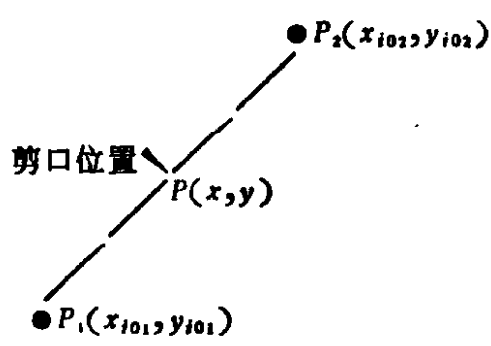

图 1

为了获得次年或多年的反应图,对于新生枝条的数量、张角、长度、抽生位置、花芽数量及 着生位置等变量,构做了一些数学仿真模型. 例如, 确定新生枝条的数量 $M$ 的模型为

$$
M=A f\left(K_{i 0}, L x_{i 0}, \alpha_{i 0}, R J K_{i 0}, S Z_{i 0}\right)+R A f,
$$

式中 $A f$ 为新生枝条数量的确定性函数, $R A f$ : 随机范围, $K_{i 0}$ ：被剪枝条生长年限， $L x_{i 0}$ : 被岦枝条类型变量， $\alpha_{i 0}$ ：被剪枝条与水平线的夹角变量， $R J K_{i 0}$ ：剪口的相对位置， $S Z_{i 0}$ : 枝 条生长势变量 
确定性函数 $A f$, 可以通过现场数据采集与已积累的经验数据, 在经统计处理后确定自 变量与因变量之间的对应关系. 为了更好地描述枝组生长随机波动情况, 再加上一个随机变 量,这样的框架可以兼容各种不同的情况（包括不同树种、品种以及生态因子、管理水平的影响 等). 随着实验数据的不断丰富, 它的精确性也会日益提高, 还可为以后构做机理模型提供可 恝的依据.

这些模型能确定修剪反应图, 并得到一些枝类比、叶果比等重要数据. 它们可以用表格等 方式在屏幕上显示或直接打印出来. 对于原始枝组生成的模型本身就是一段程序, 通过输人 有关坐标值, 就可获得相应的图形; 也可以从一根一年生枝条, 通过启发性信息的提示及有关 图形技术, 让它根据一定的生成规则逐年生长, 最后得到所欲研究的那种类型的枝组. 用同样 的方式也可按修尊者的意图生成所需要的整棵树形.

\section{二、传尊评价思维过程的模推}

在参考我国著名果树专家有关论著 ${ }^{[1-4]}$ 、安徽省苹果修韵技术资料以及广泛听取许多专家 学者意见的基础上,以一般公认的修剪规律和基本的思维模式作为主体,对枝组本身条件、周 围环境情况、修剪目的、剪法以及发枝、成花、生长势等问题作必要的说明, 提供评价依据和一 些参考性的结论; 同时设计了一个系统维护模块, 通过人机对话, 可对评价的知识、经验等内容 方便地修改、增删，由此构成一个修剪评价系统的框架. 其中的具体内容能统一的尽量统一, 不一致的地方则允许争论,保留不同意见,最终让实践去验证。

要用计算机来模拟修韵评价的一般思维过程需要经过“知识获取”的难关,工作量相当大. 首先要在不同学科之间进行沟通,取得共同语言,然后一起对修愬专家已掌握的知识、经验、规 律作进一步的开发,经过反复分析、综合、提炼才能逐步归范到计算机软件能实现的形式.例 如归纳成如下形式:

\begin{tabular}{l|c|c|c||c|c|}
\hline 枝组类型 & 环境因䋈 & 目 的 & 竘 法 & 二级因素 & 评 语 \\
\hline $1,2, \ldots, i$ & $1,2, \ldots i$ & $1,2, \ldots k$ & $1,2, \ldots l$ & $1,2, \ldots m$ & $1,2, \ldots m$ \\
\hline
\end{tabular}

前提 $\rightarrow$ 结论

图形处理和自动评价实现的机制如图 2 所示.

\section{三、软件研制情况与应用前景}

本软件是采用了结构化系统分析、系统设计与程序设计的方法即 SA、SD、SP 法，在 IBM PC/XT 微机上开发成功的,占存储容量 $400 \mathrm{k}$.

在图形处理方面, 设置了图形库和参数库,采用了图形多窗口技术将屏幕分成图形、菜单、 提示三个部分, 又利用计算机图形学中的还原、移动、旋转、摖除、组合、装配、生成、颜色变化等 各种技术处理,使一个复杂的修剪问题变得一目了然. 目前已能自动生成修剪反应图 100 多 万幅,并给出相应评价.

在实现自动评价时, 将所抽取出来的知识、经验、规则都存放到知识库中 (本软件分为一 级、二级). 选用产生式和过程这两种知识表示形式相互结合, 互为补充; 整个搜索过程采用正 向推理. 


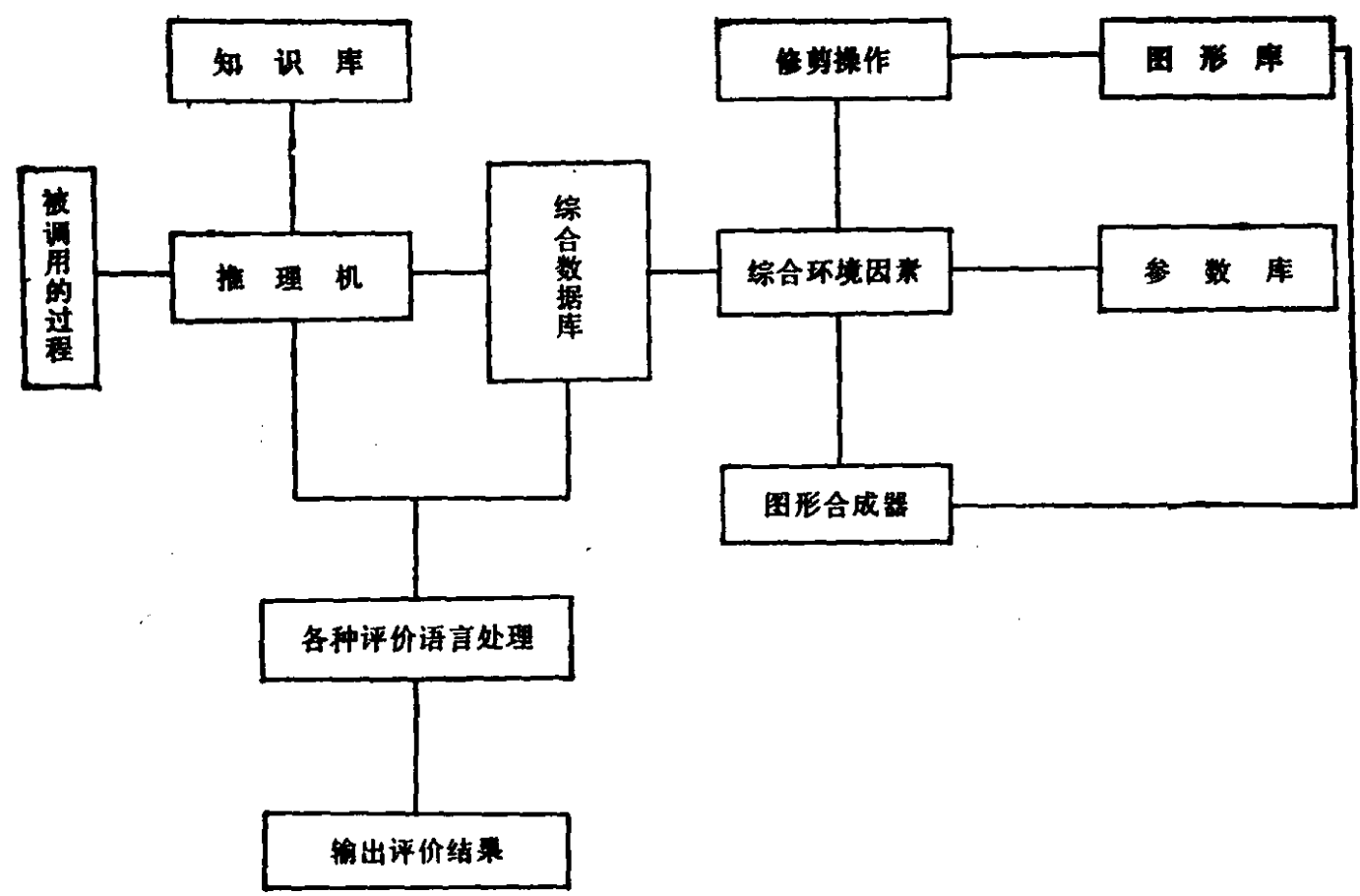

图 2 图形处理与自动评价实现的机制示意图

该软件是一个多学科进行交又研究的阶段性成果，其第一版已通过省级鉴定，用户反映良 好,认为在屏幕上进行修仿真, 可以不受时空限制, 能节省大量的人力、物力、财力和时间. 由 于不必破坏果树实体，所以不会造成任何经济损失,有独到的使用价值. 但我们的工作还是初 步的,对于修勭反应图及其评价的仿真模型还是粗线条的. 今后打算一方面不断完善本系统， 另一方面提高研究层次，采用 $\mathrm{CAD}$ 、动态规划等技术来研制对于果树修剪或栽培的群体设 计(包括矮化密植等内容)的仿真工具.

致谢: 浙江农业大学吴耕民教授,华中农业大学章文才教授，安徽省余厚敏、储晓明、冯袈、杜德裕、朱俊 杰等果树专家对本项目曾提出许多中肯意见, 上海植物生理研究所王天铎研究员对本系统的研究始终给予 热情支持,一并在此致谢.

[1]吴耕民，果时学,上海科学技术出版社, 1985,13-49,117-124,149-167.

[2] 吴光林,果对意形与海科学技术出版社, 1986,3-12,108-120,214 - 228.

[3] 河北农业大学主编,果树裁培学总论,农业出版社,北京, 1982,262-290.

[4]农学院主编,果树研究法,农业出版社, 北京, 1981,312-322. 